\title{
HYPERCONVEXITY AND NEIGHBORHOOD BASIS OF REINHARDT DOMAINS
}

\author{
Jeong-Seung LEE, Jong Jin KIM, Chun-Young OH and Sung-Hee PARK
}

(Received 27 November 2007)

\begin{abstract}
In this paper, we prove that every bounded Reinhardt domain in $\mathbb{C}^{n}$ admitting a weak Stein neighborhood basis is hyperconvex. In addition, we show that every unbounded pseudoconvex Reinhardt domain in $\mathbb{C}^{n}$ satisfying the Fu condition admits a Stein neighborhood basis.
\end{abstract}

\section{Introduction}

For a bounded domain $D$ in $\mathbb{C}^{n}$, a weak Stein neighborhood basis of $D$ is a family of pseudoconvex domains $\left\{\Omega_{k}\right\}_{k \geq 1}$ such that $\bar{D} \subset \Omega_{k}, k \in \mathbb{N}$ and $D=$ int $\bigcap_{k \in \mathbb{N}} \Omega_{k}$. Generally, for a bounded fat pseudoconvex Reinhardt domain $D$ in $\mathbb{C}^{n}$, there may not exist a weak Stein neighborhood basis of $D$. But any bounded hyperconvex Reinhardt domain $D$ has a Stein neighborhood basis which means that there is a family of pseudoconvex domains $\left\{\Omega_{k}\right\}_{k \geq 1}$ such that $\Omega_{k}$ is a domain of holomorphy and $\bar{D} \subset \Omega_{k}, k \in \mathbb{N}$ and $\bar{D}=\bigcap_{k \in \mathbb{N}} \Omega_{k}$ (see [9]). Note that every fat domain having a Stein neighborhood basis always has a weak Stein neighborhood basis. For example, the Hartogs triangle is a bounded fat pseudoconvex Reinhardt domain in $\mathbb{C}^{2}$ but it does not allow a weak Stein neighborhood basis.

It is worth noting that every pseudoconvex domain containing the closure of the Hartogs triangle also contains the closure of the unit bidisc [4]. Note that the unit bidisk is hyperconvex, while the Hartogs triangle is not so. By observing this phenomenon, in [9] (or [5]), the authors showed that if any bounded fat Reinhardt domain $D$ in $\mathbb{C}^{n}$ has a Stein neighborhood basis, then $D$ is hyperconvex. In this paper, we shall show that the same result still holds under a weaker condition. Namely, every bounded Reinhardt domain in $\mathbb{C}^{n}$ having a weak Stein neighborhood basis is hyperconvex.

On the other hand, it is natural to ask whether an unbounded Reinhardt domain having a Stein neighborhood basis can be hyperconvex. Unfortunately, it is impossible because any hyperconvex Reinhardt domain is bounded [13]. Now we are interested in the following: Under what condition will an unbounded Reinhardt domain admit a Stein neighborhood basis? As a partial answer to that question, we shall show that there exists a class of unbounded pseudoconvex Reinhardt domains admitting a Stein neighborhood basis.

2000 Mathematics Subject Classification: Primary 32A07, 32T05.

Keywords: Reinhardt set; hyperconvexity; weak Stein neighborhood basis; Stein neighborhood basis.

(c) 2009 Faculty of Mathematics, Kyushu University 


\section{Preliminaries}

A set $D$ in $\mathbb{C}^{n}$ is said to be Reinhardt whenever

$$
\left(z_{1}, \ldots, z_{n}\right) \in D \Longrightarrow\left(e^{i \theta_{1}} z_{1}, \ldots, e^{i \theta_{n}} z_{n}\right) \in D, \quad \theta_{i} \in \mathbb{R}, 1 \leq i \leq n .
$$

For $j=1, \ldots, n$, we set $V_{j}:=\left\{z \in \mathbb{C}^{n}: z_{j}=0\right\}, V:=\bigcup_{1 \leq j \leq n} V_{j}$, and $D_{*}:=D \backslash V$. Denote $V_{I}:=V_{j_{1}} \cap \cdots \cap V_{j_{k}}$ where $I=\left\{j_{1}, \ldots, j_{k}\right\} \subset\{1, \ldots, n\}$. Given a Reinhardt subset $D$ of $\mathbb{C}^{n}$, we denote by $\log \left(D_{*}\right)$ its logarithmic image, i.e.

$$
\log \left(D_{*}\right):=\left\{\left(\log \left|z_{1}\right|, \ldots, \log \left|z_{n}\right|\right) \in \mathbb{R}^{n}:\left(z_{1}, \ldots, z_{n}\right) \in D_{*}\right\} .
$$

For a domain $D$ in $\mathbb{C}^{n}$, we denote by $\mathcal{E}(D)$ the envelope of holomorphy of $D$, i.e.

$$
\mathcal{E}(D):=\text { int } \bigcap_{\substack{D \subset U \subset \mathbb{C}^{n} \\ U \text { is a pseudoconvex } \\ \text { domain }}} U .
$$

Observe that $\mathcal{E}(D)$ is the smallest domain of holomorphy containing $D$ and if $D$ is a Reinhardt domain, then $\mathcal{E}(D)$ is also a Reinhardt domain in $\mathbb{C}^{n}$ (see [2]). Therefore, the envelope of holomorphy of any Reinhardt domain in $\mathbb{C}^{n}$ is a pseudoconvex Reinhardt domain. Note that if $D \Subset G$, then $\mathcal{E}(D) \Subset \mathcal{E}(G)$ (see [12]). For more information regarding envelopes of holomorphy we refer the reader to $[6,7]$.

We denote by $\mathcal{P} \mathcal{S H}(D)$ the set of all plurisubharmonic functions on $D$, and by $P(z, \varepsilon)$ the open polydisc in $\mathbb{C}^{n}$ of multiradius $\varepsilon=\left(\varepsilon_{1}, \ldots, \varepsilon_{n}\right), \varepsilon_{j}>0$, centered at $z \in \mathbb{C}^{n}$.

Definition 2.1. A domain $D$ in $\mathbb{C}^{n}$ is hyperconvex if there exists a negative continuous plurisubharmonic exhaustion function $u$ on $D$, i.e. $u \in(\mathcal{C} \cap \mathcal{P S \mathcal { H }})(D), u<0$ on $D$, and $\lim _{z \rightarrow \partial D} u(z)=0$ (here and below $\infty \in \partial D$ if $D$ is unbounded). Such a function $u$ is sometimes called a continuous plurisubharmonic barrier on $D$.

The notion of hyperconvexity is an important concept in pluripotential theory. For hyperconvexity, it is enough to have a weak plurisubharmonic barrier for every boundary point, as follows.

Proposition 2.2. [1] A domain $D$ in $\mathbb{C}^{n}$ is hyperconvex if and only if there is a weak plurisubharmonic barrier at every point $\xi \in \partial D$, i.e. there exists a negative plurisubharmonic function $\psi$ on $D$ such that $\lim _{z \rightarrow \xi} \psi(z)=0$.

This notion is perhaps most clearly stated by Błocki [1]. The point $\xi$ as in Proposition 2.2 is called a barrier point for $D$.

Let us recall the following geometrical criterion for a Reinhardt domain in $\mathbb{C}^{n}$ to be pseudoconvex.

Proposition 2.3. $[6,12]$ Let $D$ be a Reinhardt domain in $\mathbb{C}^{n}$. Then the following conditions are equivalent:

(1) D is pseudoconvex;

(2) $\log \left(D_{*}\right)$ is convex, and if $D \cap V_{j} \neq \varnothing$ for some $j \in\{1, \ldots, n\}$, then

$$
\left(z_{1}, \ldots, z_{j}, \ldots, z_{n}\right) \in D \Longrightarrow\left(z_{1}, \ldots, \lambda z_{j}, \ldots, z_{n}\right) \in D, \quad|\lambda| \leq 1 .
$$


Let us mention that the previous criterion plays an important role in the study of the pseudoconvexity of Reinhardt domains.

For an index set $I=\left\{j_{1}, \ldots, j_{k}\right\} \subset\{1, \ldots, n\}$, we define the canonical projection $\pi_{I}: \mathbb{C}^{n} \longrightarrow \mathbb{C}^{n-k}$ by $\pi_{I}(z):=\left(z_{m_{1}}, \ldots, z_{m_{n-k}}\right)$, where $z=\left(z_{1}, \ldots, z_{n}\right) \in \mathbb{C}^{n},\left\{m_{1}, \ldots\right.$, $\left.m_{n-k}\right\}:=\{1, \ldots, n\} \backslash I$. The following result is easily obtained.

Proposition 2.4. [13] Let D be a pseudoconvex Reinhardt domain in $\mathbb{C}^{n}$ and $D \cap V_{I} \neq \varnothing$ where $I=\left\{j_{1}, \ldots, j_{k}\right\} \subset\{1, \ldots, n\}$. Then $\pi_{I}(D)$ is also a pseudoconvex Reinhardt domain in $\mathbb{C}^{n-k}$.

Definition 2.5. For a bounded domain $D \subset \mathbb{C}^{n}$ we say that:

- $D$ has a weak Stein neighborhood basis if

$$
D=\operatorname{int} \bigcap_{\begin{array}{c}
\bar{D} \subset U \subset \mathbb{C}^{n} \\
U \text { is a domain } \\
\text { of holomorphy }
\end{array}} U ;
$$

- $D$ admits a Stein neighborhood basis if

$$
\bar{D}=\bigcap_{\substack{\bar{D} \subset U \subset \mathbb{C}^{n} \\ U \text { is a domain } \\ \text { of holomorphy }}} U .
$$

Notice that any domain in $\mathbb{C}^{n}$ admitting a (weak) Stein neighborhood basis is a domain of holomorphy. Obviously, the second notion from the above definitions plus a fatness condition implies the first one. For details, we refer to e.g. [7]. Furthermore, there is a bounded pseudoconvex domain that has a weak Stein neighborhood basis, but not a Stein neighborhood basis (see e.g. [11]).

\section{Hyperconvexity of bounded Reinhardt domains}

Before stating our main result let us make some preparations.

Proposition 3.1. (Cf. [3]) Let D be a bounded pseudoconvex Reinhardt domain in $\mathbb{C}^{n}$. Then, for every point $\xi$ in $(\partial D) \backslash V$, there exists a weak plurisubharmonic barrier $u$ on $D$ at $\xi$.

Combining Proposition 2.2 and Proposition 3.1, we obtain the following result.

Corollary 3.2. Let $D$ be a bounded pseudoconvex Reinhardt domain in $\mathbb{C}^{n}$. D is hyperconvex if and only if, for every point $\xi \in(\partial D) \cap V$, there exists a weak plurisubharmonic barrier $u$ on $D$ at $\xi$.

In the following we shall often use the following geometrical characterization of hyperconvex Reinhardt domains due to P. Pflug and W. Zwonek (see [13]).

TheOREM 3.3. [13] Let D be a pseudoconvex Reinhardt domain in $\mathbb{C}^{n}$. Then the following conditions are equivalent:

(1) $D$ is hyperconvex;

(2) $D$ is bounded and if $\bar{D} \cap V_{j} \neq \emptyset$ then $D \cap V_{j} \neq \emptyset$ for $1 \leq j \leq n$. 
The second condition in (2) is called the ' $F u$-condition'. It is an immediate consequence of Theorem 3.3 that every bounded pseudoconvex Reinhardt domain $D$ with $\bar{D} \cap V=\emptyset$ is hyperconvex.

To verify our main theorem, we need the following results.

Lemma 3.4. Let $D$ be a bounded domain in $\mathbb{C}^{n}$ having a weak Stein neighborhood basis $\left\{U_{\alpha}\right\}_{\alpha \geq 1}$. Then $D$ is fat.

LEMMA 3.5. If $D$ is a bounded domain in $\mathbb{C}^{n}$ having a weak Stein neighborhood basis, then $D$ has a weak Stein neighborhood basis $\left\{W_{\alpha}\right\}_{\alpha \geq 1}$ such that $\overline{W_{\alpha+1}} \subset W_{\alpha}$ for $\alpha \in \mathbb{N}$.

Lemma 3.6. Let $D$ be a bounded Reinhardt domain in $\mathbb{C}^{n}$ having a weak Stein neighborhood basis. Then there exists a weak Reinhardt Stein neighborhood basis $\left\{W_{j}\right\}_{j \geq 1}$ of $D$ such that $\overline{W_{j+1}} \subset W_{j}$ for $j \in \mathbb{N}$.

Proof. By Lemma 3.5, we may assume that $D$ has a weak Stein neighborhood basis $\left\{U_{j}\right\}_{j \geq 1}$ such that $\overline{U_{j+1}} \subset U_{j}$ for $j \in \mathbb{N}$ and each $U_{j}$ is bounded. Since $D$ is a bounded Reinhardt domain and $\bar{D} \subset U_{j}$ for $j \in \mathbb{N}$, we can choose a strictly decreasing sequence $\left\{r_{j}\right\}_{j \geq 1}$ of positive numbers with $P\left(z,\left(r_{j}, \ldots, r_{j}\right)\right) \subset U_{j}$ for any $z \in \partial D$, and for each $z=\left(z_{1}, z_{2}, \ldots, z_{n}\right) \in \partial D$ and $j \geq 1$, put $A_{z}(j):=A_{z_{1}}(j) \times \cdots \times A_{z_{n}}(j)$, where

$$
A_{z_{s}}(j):=\left\{\begin{array}{ll}
\left\{\zeta \in \mathbb{C}: \max \left\{0,\left|z_{s}\right|-r_{j}\right\}<|\zeta|<\left|z_{s}\right|+r_{j}\right\} & \left(z_{s} \neq 0\right) \\
\left\{\zeta \in \mathbb{C}:|\zeta|<r_{j}\right\} & \left(z_{s}=0\right)
\end{array} \quad(1 \leq s \leq n) .\right.
$$

Then each $A_{z}(j)(z \in \partial D)$ is a Reinhardt domain with $A_{z}(j) \subset U_{j}$, and also $M_{j}:=D \cup$ $\left(\bigcup_{z \in \partial D} A_{z}(j)\right)$ is a Reinhardt domain contained in $U_{j}$. Moreover,

$$
D \Subset M_{j+1} \Subset M_{j} \subset U_{j}(j \geq 1), \quad D=\operatorname{int} \bigcap_{j \geq 1} U_{j}=\operatorname{int} \bigcap_{j \geq 1} M_{j} .
$$

We proceed by setting $W_{j}:=\mathcal{E}\left(M_{j}\right)$ for $j \geq 1$, and then we get $\overline{W_{j+1}} \subset W_{j}$ by virtue of [2] and [11].

Now we are going to state and prove our main result.

THEOREM 3.7. A bounded Reinhardt domain D in $\mathbb{C}^{n}$ having a weak Stein neighborhood basis is hyperconvex.

Proof. By virtue of Lemma 3.6, we can take a family of bounded pseudoconvex Reinhardt domains $\left\{U_{k}\right\}_{k \geq 1}$ such that $\overline{U_{k+1}} \subset U_{k}$ for $k \geq 1$ and

$$
D=\operatorname{int} \bigcap_{k \geq 1} U_{k}
$$

Let $a \in(\partial D) \cap V$. We shall first show that $a \neq 0$. Suppose that the origin 0 is contained in $\partial D$, and take a point $p=\left(p_{1}, \ldots, p_{n}\right) \in D_{*}$. Then 0 and $p$ are contained in $U_{k}$ for any $k \geq 1$. Since $p \notin V$, one has $p_{i} \neq 0$ for $i=1, \ldots, n$. Put

$$
E:=\left\{\left(z_{1}, \ldots, z_{n}\right):\left|z_{i}\right| \leq\left|p_{i}\right|, i=1, \ldots, n\right\} .
$$

By Proposition 2.3, one has $E \subset U_{k}$ for any $k \geq 1$, and so $E \subset \bigcap_{k \geq 1} U_{k}$. Because the origin 0 is an interior point of $E$, one has

$$
0 \in \operatorname{int} E \subset \operatorname{int} \bigcap_{k \geq 1} U_{k}=D,
$$


which is a contradiction to our assumption. Therefore, we have $a \neq 0$. Now, suppose that $a=$ $\left(a_{1}, \ldots, a_{n-j}, 0, \ldots, 0\right)$ and $a_{1} \cdots a_{n-j} \neq 0$ for some $0 \leq j<n$. We are going to show that $\pi(a) \in \partial \pi(D)$, where $\pi:=\pi_{\{n-j+1, \ldots, n\}}$. Notice that $\pi(a) \in \pi(\bar{D}) \subset \overline{\pi(D)}$. Assume that $\pi(a) \in \pi(D)$. Then there is a point $a^{\prime \prime} \in \mathbb{C}^{j}$ such that $a^{*}:=\left(\pi(a), a^{\prime \prime}\right) \in D$. Moreover, we can choose a Reinhardt open neighborhood $\mathcal{N}=\mathcal{N}\left(a^{*}\right)$ in $D$. Fix $m \geq 1$. Observe that $a \in \partial D \subset U_{m}$. Hence we have $U_{m} \cap V_{k} \neq \varnothing$ for every $n-j+1 \leq k \leq n$. But since each $U_{m}$ is a pseudoconvex Reinhardt domain, it follows from Proposition 2.3 that

$$
W:=\left\{\begin{array}{l|l}
\left(z, \lambda_{1} w_{1}, \ldots, \lambda_{j} w_{j}\right) \in \mathbb{C}^{n-j} \times \mathbb{C}^{j} & \begin{array}{l}
\left(z, w_{1}, \ldots, w_{j}\right) \in \mathcal{N}, \\
\left|\lambda_{k}\right| \leq 1, k=1, \ldots, j
\end{array}
\end{array}\right\} \subset U_{m} .
$$

In particular, $a \in W$. But since $m \geq 1$ is arbitrary, we get that

$$
a \in W=\operatorname{int} W \subset \operatorname{int} \bigcap_{m \geq 1} U_{m}=D,
$$

which is a contradiction to the fact that $a \in \partial D$. Consequently, it must be $\pi(a) \notin \pi(D)$. Hence $\pi(a) \in \partial \pi(D)$. On the other hand, since $\pi(D)$ is a bounded pseudoconvex Reinhardt domain, Lemma 3.1 implies that there exists a weak plurisubharmonic barrier $u$ at $\pi(a)$ in $\pi(D)$. Therefore, the function $u \circ \pi$ is a weak plurisubharmonic barrier at $a$ in $D$, and so we are done by Corollary 3.2.

Now we are going to discuss related examples. To do this, for every $\alpha>0$ we define the (possibly multivalued) function

$$
\lambda^{\alpha}:= \begin{cases}e^{\alpha(\ln |\lambda|+i \arg \lambda)} & \left(\lambda \in \mathbb{C}_{*}\right), \\ 0 & (\lambda=0),\end{cases}
$$

and for every set $D$ in $\mathbb{C}^{n}$ we denote

$$
D^{\alpha}:=\left\{\left(z_{1}^{\alpha}, \ldots, z_{n}^{\alpha}\right):\left(z_{1}, \ldots, z_{n}\right) \in D\right\} .
$$

To proceed with our discussion we need the following auxiliary result.

Proposition 3.8. [9] Let $D$ be a bounded hyperconvex Reinhardt domain in $\mathbb{C}^{n}$. Then $\left\{D^{\alpha}\right\}_{\alpha>1}$ is a Stein neighborhood basis of $D$.

In fact, its proof in [9] essentially guarantees that the previous statement without the assumption of boundedness is still true. For details, we would like to give the proof in the next section.

Example 3.9. (a) Since every pseudoconvex domain containing $\bar{\triangle}_{H}$ also contains the unit bidisk, $\triangle_{H}$ does not have a weak Stein neighborhood basis. Note that $0 \in \partial \triangle_{H}$.

(b) The domain $G:=\{(z, w) \in \mathbb{D}: 0<|w|-|z|<1 / 2\}$ is not pseudoconvex. Moreover, $\mathcal{E}(G)=\triangle_{H}$ does not have a weak Stein neighborhood basis. Observe that the origin is a boundary point of $\mathcal{E}(G)$.

Consequently, we obtain the following characterization of hyperconvexity in Reinhardt domains.

THEOREM 3.10. Let D be a bounded Reinhardt domain in $\mathbb{C}^{n}$. Then the following conditions are equivalent. 
(1) D is hyperconvex.

(2) D is strictly hyperconvex.

(3) D is fat and has a Stein neighborhood basis.

(4) D admits a weak Stein neighborhood basis.

For the notion mentioned in (2) we refer to [10] (or [5]).

Remark 3.11. (a) In Theorem 3.10, the implications (1) $\Longleftrightarrow(2) \Longleftrightarrow$ (3) were proved by Le Mau Hai et al [5]. In their proof, for the implication (3) $\Longrightarrow(1)$, the fatness of the given Reinhardt domain played an important role.

(b) Notice that the implication (3) $\Longrightarrow$ (4) is obvious but the converse is, in general, not true. As already mentioned, there is a bounded pseudoconvex domain that has a weak Stein neighborhood basis, but does not have a Stein neighborhood basis (cf. [11]). In this paper, we proved that a bounded Reinhardt domain is hyperconvex under an apparently weaker assumption.

Let us close this section with the following statement.

COROllary 3.12. Any bounded Reinhardt domain in $\mathbb{C}^{n}$ admitting a weak Stein neighborhood basis always has a Stein neighborhood basis.

\section{Remarks on unbounded Reinhardt domains}

In this section we ask whether an unbounded Reinhardt domain has a Stein neighborhood basis? From now on, we are going to give a class of pseudoconvex Reinhardt domains for which the answer to the previous question is always positive. To see this, we first need the following lemma.

Lemma 4.1. Any pseudoconvex Reinhardt domain in $\mathbb{C}^{n}$ satisfying the Fu condition is fat.

Proof. Suppose that $D \subset \mathbb{C}^{n}$ is a pseudoconvex Reinhardt domain but not fat. Then there is a point $z^{0}=\left(z_{1}^{0}, \ldots, z_{n}^{0}\right) \in($ int $\bar{D}) \backslash D$. First, assume that $z^{0} \notin V$. Observe that

$$
\log \left|z^{0}\right| \in \log \left[(\operatorname{int} \bar{D})_{*}\right]=\log \left(D_{*}\right) .
$$

Here, in the above equality, we have used the fact that the convexity of $\log \left(D_{*}\right)$ implies that $\log \left[(\text { int } \bar{D})_{*}\right] \subset \log \left(D_{*}\right)$. Hence we have $z^{0} \in D$, which is a contradiction. Next, suppose that $z^{0} \in V_{\mu}$ for some $\mu \in\{1,2, \ldots, n\}$. Since $D$ is a pseudoconvex Reinhardt domain, there are only two cases:

(i) if there does not exist a point $\left(z_{1}, \ldots, z_{n}\right) \in D$ such that $z_{\mu} \neq 0$ and $z_{v}=z_{v}^{0}$ for $v \neq \mu$, then $D$ is not connected, which is a contradiction;

(ii) if there does not exist a point $z \in D$ with $z \in V_{\mu}$, that is, $D \cap V_{\mu}=\emptyset$, the domain $D$ can no longer satisfy the Fu condition.

From now on, similar to Definition 2.5, we say that an unbounded pseudoconvex Reinhardt domain $D$ in $\mathbb{C}^{n}$ has a Stein neighborhood basis if $D$ is fat and

$$
\bar{D}=\bigcap_{\substack{\bar{D} \subset U \subset \mathbb{C}^{n} \\ U \text { is a domain } \\ \text { of holomorphy }}} U .
$$


THEOREM 4.2. If $D \subset \mathbb{C}^{n}$ is an unbounded pseudoconvex Reinhardt domain satisfying the Fu condition, then $D$ has a Stein neighborhood basis.

The following proof is modified from an argument in the original one for Proposition 3.8.

Proof. Assume by changing coordinates if necessary that $(1, \ldots, 1) \in D$. It suffices to prove that $\left\{D^{\alpha}\right\}_{\alpha>1}$ is a Stein neighborhood basis for $D$. We first prove that for $\alpha>1$ the domain $D^{\alpha}$ is pseudoconvex Reinhardt. Fix $\alpha>1$. If $\left(z_{1}, \ldots, z_{n}\right) \in D$ and $\lambda_{i} \in \mathbb{C}$ with $\left|\lambda_{i}\right|=1$ where $i \in\{1, \ldots, n\}$, then $\left(\lambda_{1}^{1 / \alpha} z_{1}, \ldots, \lambda_{n}^{1 / \alpha} z_{n}\right) \in D$ and also $\left(\lambda_{1} z_{1}^{\alpha}, \ldots, \lambda_{n} z_{n}^{\alpha}\right) \in D^{\alpha}$. Hence $D^{\alpha}$ is Reinhardt. Observe that $\log \left(D_{*}\right)$ is convex and

$$
\log \left[\left(D^{\alpha}\right)_{*}\right]=\alpha \log \left(D_{*}\right),
$$

so the set $\log \left[\left(D^{\alpha}\right)_{*}\right]$ is also convex. Assume now that $D^{\alpha} \cap V_{j} \neq \varnothing$ for some $j \in$ $\{1, \ldots, n\}$. Then it is clear that $D \cap V_{j} \neq \varnothing$, and because of the pseudoconvexity of $D$ one has

$$
\left(z_{1}, \ldots, z_{j}, \ldots, z_{n}\right) \in D \Longrightarrow\left(z_{1}, \ldots, \lambda^{1 / \alpha} z_{j}, \ldots, z_{n}\right) \in D, \quad|\lambda| \leq 1 .
$$

So, by Proposition 2.3, we get the pseudoconvexity of $D^{\alpha}$.

Next, to show that $\bar{D} \subset \bigcap_{\alpha>1} D^{\alpha}$, let $a=\left(a_{1}, \ldots, a_{n}\right) \in \bar{D}$ and $\alpha>1$. We shall discuss the following three cases.

(c1) The case $a \notin V$. Because of the convexity of $\log \left(D_{*}\right)$ one has $\overline{\log \left(D_{*}\right)} \subset \alpha \log \left(D_{*}\right)$. Hence, by $(\dagger),\left(\log \left|a_{1}\right|, \ldots, \log \left|a_{n}\right|\right) \in \log \left(D^{\alpha}\right)_{*}$. But since $D^{\alpha}$ is Reinhardt, we have $a \in D^{\alpha}$.

(c2) The case $a=(0, \ldots, 0)$. Since $\bar{D} \cap V_{j} \neq \varnothing$ for any $j \in\{1, \ldots, n\}$, the Fu condition for $D$ implies that $D \cap V_{j} \neq \varnothing$ for any $j \in\{1, \ldots, n\}$. By Proposition 2.3, we get $a \in D \subset D^{\alpha}$.

(c3) The case $a \in V \backslash\{0\}$. Without loss of generality, we may assume that $a=$ $\left(0, \ldots, 0, a_{k+1}, \ldots, a_{n}\right)$, where $a_{k+1} \cdots a_{n} \neq 0,1 \leq k<n$. Denote $\pi:=\pi_{\{1, \ldots, k\}}$. Then $\pi(D)$ is a pseudoconvex Reinhardt domain in $\mathbb{C}^{n-k}$ and

$$
\pi(a) \notin \bigcup_{j=k+1}^{n} \pi\left(V_{j}\right), \quad \pi(a) \in \pi(\bar{D}) \subset \overline{\pi(D)} .
$$

This is a similar situation as in the case (c1). Hence, we obtain that $\pi(a) \in[\pi(D)]^{\alpha}=$ $\pi\left(D^{\alpha}\right)$. On the other hand, because of the Fu condition for $D$, for each $j \in\{1, \ldots, k\}$ one has $D \cap V_{j} \neq \varnothing$ and so $D^{\alpha} \cap V_{j} \neq \varnothing$. Thus it follows from Proposition 2.3 that $a \in D^{\alpha}$, as desired.

Finally, it remains to see that $\bigcap_{\alpha>1} D^{\alpha} \subset \bar{D}$. For this, let $a \in \bigcap_{\alpha>1} D^{\alpha}$. Notice that the convexity of $\log \left(D_{*}\right)$ implies that

$$
\bigcap_{\alpha>1} \log \left[\left(D^{\alpha}\right)_{*}\right]=\bigcap_{\alpha>1} \alpha \log \left(D_{*}\right)=\overline{\log \left(D_{*}\right)} .
$$

Now, we are going to discuss the following three cases.

(k1) The case $a \notin V$. Note that $\left(\log \left|a_{1}\right|, \ldots, \log \left|a_{n}\right|\right) \in \log \left[\left(D^{\alpha}\right)_{*}\right]$ for any $\alpha>1$. By (†), it follows that $\log |a| \in \overline{\log \left(D_{*}\right)}=\log \left[(\bar{D})_{*}\right]$ and also $a \in \bar{D}$.

(k2) The case $a=(0, \ldots, 0)$. Since $0 \in D^{\alpha}$ for any $\alpha>1$, one has $0 \in D$ and so $0 \in \bar{D}$. 
(k3) The case $a \in V \backslash\{0\}$. We may assume without loss of generality that $a=$ $\left(0, \ldots, 0, a_{k+1}, \ldots, a_{n}\right)$, where $a_{k+1} \cdots a_{n} \neq 0,1 \leq k<n$. By the case (k1) one has $\pi(a) \in \overline{\pi(D)}$. Observe the following.

(i) The case $\pi(a) \in \pi(D)$. Let $j \in\{k+1, \ldots, n\}$. Since $D^{\alpha} \cap V_{j} \neq \emptyset$, one has $D \cap V_{j} \neq \emptyset$. By Theorem 3.10, we get $a \in D$.

(ii) The case $\pi(a) \in \partial(\pi(D))$. Suppose that $a \notin \bar{D}$, then there is an open polydisc

$$
\mathbb{P}(a):=\left\{\left(z_{1}, \ldots, z_{n}\right) \in \mathbb{C}^{n}: \begin{array}{l}
\left|z_{1}\right|<\varepsilon_{1}, \ldots,\left|z_{k}\right|<\varepsilon_{k}, \\
\left|z_{k+1}-a_{k+1}\right|<\varepsilon_{k+1}, \ldots,\left|z_{n}-a_{n}\right|<\varepsilon_{n}
\end{array}\right\}
$$

such that $\mathbb{P}(a) \subset \mathbb{C}^{n} \backslash \bar{D}$. Put $\mathbb{P}(\pi(a)):=\pi(\mathbb{P}(a))$. Because $\mathbb{P}(\pi(a)) \cap \pi(D)=$ $\emptyset$, we obtain $\pi(a) \notin \partial \pi(D)$, which is a contradiction. Thus, $a \in \bar{D}$.

Remark 4.3. Theorem 3.3 shows that every hyperconvex Reinhardt domain is bounded. So, we would like to mention that any unbounded pseudoconvex Reinhardt domain cannot be hyperconvex although it has a Stein neighborhood basis.

Finally, we shall give such an example.

Example 4.4. Let $E:=\left\{\left(z_{1}, z_{2}\right) \in \mathbb{C}^{2}:\left|z_{1}\right|<C\right\}$. Clearly, $E$ is a unbounded pseudoconvex Reinhardt domain satisfying the Fu condition. Now, if we set $U_{n}:=\left\{\left(z_{1}, z_{2}\right) \in \mathbb{C}^{2}:\left|z_{1}\right|<\right.$ $C+1 / n\}$ for $n \in \mathbb{N}$, then $\left\{U_{n}\right\}_{n \geq 1}$ is a Stein neighborhood basis of $E$, but $E$ is not a hyperconvex.

Acknowledgements. The authors would like to express our gratitude to Professor Peter Pflug. His valuable comments made on an earlier version of this paper led to significant improvements in the final manuscript. We are also grateful for the referee for valuable suggestions. While preparing this paper for publication, the authors heard about a related work that was introduced to the literature [8] which was published recently.

\section{REFERENCES}

[1] Z. Błocki. The complex Monge-Ampère operator in hyperconvex domains. Ann. Scuola Norm. Pisa Cl. Sci. 4 (1996), 721-747.

[2] R. Carmignani. Envelopes of holomorphy and holomorphic convexity. Trans. Amer. Math. Soc. 179 (1973), 415-430.

[3] M. Carlehed, U. Cegrell and F. Wikström. Jensen measure, hyperconvexity and boundary behaviour of the pluricomplex Green functions. Ann. Polon. Math. 71 (1999), 87-103.

[4] J. E. Fornaess and B. Stensønes. Lectures on Counterexamples in Several Complex Variables (Mathematical Notes, 33). Princeton University Press, Princeton, NJ, 1987.

[5] Le Mau Hai, Nguyen Quang Dieu, and Nguyen Huu Tuyen. Some properties of Reinhardt domains. Ann. Polon. Math. 82 (2003), 203-217.

[6] P. Jakóbczak and M. Jarnicki. Lectures on holomorphic functions of several complex variables. PS File at http://www.im.uj.edu.pl/ jarnicki/mjp.htm (2001).

[7] M. Jarnicki and P. Pflug. Extension of Holomorphic Functions (de Gruyter Expositions in Mathematics, 34). Walter de Gruyter, Berlin, 2000.

[8] M. Jarnicki and P. Pflug. First Steps in Several Complex Variables: Reinhardt Domains (EMS Textbooks in Mathematics). European Mathematical Society, Zürich, 2008.

[9] Nguyen Quang Dieu and Le Mau Hai. Some remarks about Reinhardt domains in $\mathbb{C}^{n}$. Illinois J. Math. 47 (2003), 699-708. 
[10] S. Nivoche. The pluricomplex Green function, capacitative notions, and approximation problems in $\mathbb{C}^{n}$. Indiana Univ. Math. J. 44 (1995), 489-510.

[11] B. Stensøness. Stein Neighborhoods. Math. Z. 195 (1987), 433-436.

[12] V. Vladimirov. Methods of the Theory of Several Complex Variables. Russian Moskau, 1964 (Engl. transl., Cambridge, MA, London, 1996).

[13] W. Zwonek. Completeness, Reinhardt domains and the method of Complex geodesics in the theory of invariants functions. Diss. Math. 388 (2000), 1-103.

Jeong-Seung Lee and Jong Jin Kim

Department of Mathematics, and Institute of Pure and Applied Mathematics

Chonbuk National University

Jeonju, Jeonbuk 561-756

Republic of Korea

(E-mail:leejs0724@chonbuk.ac.kr,jjkim@chonbuk.ac.kr)

Chun-Young Oh

Department of Applied Mathematics

Chonnam National University

Yeosu, Chonnam 550-756

Republic of Korea

(E-mail: cyoh@chonnam.ac.kr)

Sung-Hee Park

Department of Mathematical Education

Jeonju University

Jeonju, Jeonbuk 560-759

Republic of Korea

(E-mail: wshpark@jj.ac.kr) 